\title{
Lithium audit in the Scottish Borders
}

\author{
John R. Taylor and lan G. Dewar
}

\begin{abstract}
This paper describes the process of lithium oudit at Dincletion Hoeplital, showing that improvements in clinical practice can be achioved and maintained by implementation of the audif cycto. Even our small oudit involved ofl of the medical stafl and several other proteselonal groups and we found this mulfidisciplinary cpproach encouraged change.
\end{abstract}

Audit is a process of change. Our paper describes the process of clinical audit of lithium in Borders Region.

In 1988 in co-operation with the biochemistry department, a lithium recall scheme for the Scottish Borders was established using manual cards. This has successfully ensured that all patients on lithium in the Borders have annual lithium levels, renal and thyroid function tests done by local GPs. The dispersed rural nature of our service and its routine home assessment of patients has previously been described by Jones (1987). There were 148 people on lithium in a population of 104,000 in October 1992. This $0.14 \%$ point prevalence is similar to $0.13 \%$ found by Kehoe \& Mander (1992).

Lithium prescribing practices would appear to be a good area to employ medical audit. The article by Aronson \& Reynold (1992) in the British Medical Journal and the correspondence that followed it showed that even among the experts there are large areas of disagreement about what is good practice. Against such a background it was decided to set local standards for lithium practices for in-patients at our regular medical audit meeting. Even if national guidelines had been readily avallable we feel that local protocols should still have been developed as recommended by the interim report of the Clinical resource and Audit Group (1992). Universal use of national standards, while improving conformity, risks local practitioners losing the sense of involvement gained from setting and implementing their own standards. This may partly explain why guidelines from conferences often do not affect clinical practice unless other factors prompting change are present (Haines \& Feder. 1992).

\section{The study}

Three standards were agreed at clinical audit meetings involving both the local biochemist and pharmacist: all lithium prescriptions to specify the trade name and whether lithium carbonate or lithium citrate is being prescribed; serum lithium levels to be estimated at least at three monthly intervals in line with current British National Formulary recommendations; and all patients receiving lithium to be given a lithium card.

After the first survey doctors at the audit meeting received feedback on their poor performance in lithium prescription and the pharmacist offered to provide all wards with lithium cards and check with nursing staff that patients had received them. These two interventions completed the first full cycle of the audit circle.

Two further surveys were completed to confirm that a change in clinical practice had taken place and was being maintained.

\section{Findings}

The initial survey showed that 14 out of 15 in-patients met the standard for lithium estimations but none met either of the other two standards. The subsequent two repeat surveys showed large improvements in our lithium prescribing practices. By the third survey 12 out of 14 in-patients met the standard for lithium estimations and lithium cards (except for one patient with learning difficulty who was not given a card).

\section{Comment}

The first survey just after the introduction of the agreed standards showed deficiencies in prescribing practices and the supply of written information to patients receiving lithium. Both areas had improved by the time of the second survey three months later but it was not until the last survey that the standard for lithium prescriptions was met. Despite some disagreement between authoritative sources as to the correct prescribing and monitoring practices in the treatment of patients with lithium, it was surprisingly simple to reach a consensus to define local protocols for lithium audit. We found implementing the 'audit cycle' of observing practice, comparing practice with standards, and 
intervening to improve practice, was a powerful way of producing change and has led to a marked improvement in several important areas of lithium practice.

\section{Conclusions}

Clinical audit of lithium prescribing practices has been simple but effective. It has induced valuable change in our own practices and could probably do so in other areas with a larger patient group.

A multidisciplinary approach, involving psychiatrists, nurses, the pharmacist and biochemist, has been valuable in our experience with lithium practices to maximise the potential of audit to effect significant changes in the way we practice. Notably after defining our standards, the most immediate and complete improvements were made by nursing staff in the wards by supplying appropriate information in the form of cards to the relevant patients and also by the pharmacy by ensuring that defined wards had cards in stock and that patients had received them.

Furthermore, additional improvements in the future depend on close liaison with other professionals. Although psychiatrists can effect change on lithium treatment practices for in-patients, the larger number of patients in the community receiving lithium may often have closer contact with other professionals. Consequently multidisciplinary approaches are likely to be vital in positively influencing practices for this larger group.

\section{Acknowledgements}

We would like to thank Dr David Usher, Clinical Biochemist, Mr Ian Barr, Pharmacist, and all the medical and nursing staff at Dingleton Hospital.

\section{References}

ARONSON, J.K. \& REYNOLD D.J.M. (1992) Lithium. Brttish Medial Journal, S08, 1273-1276.

Clinical Resource AND AUDIT Group (1992) Clinical Outcome Measures. Executtve Summary of An Intertm Report (Chairman J. Kyle). Edinburgh: Scottish Office.

HAINES, A. \& FEDER, G. (1992) Guidence on guidelines. British Medical Journal, 305, 785-786.

JONES, D. (1987) Community peychiatry in the borders. In Creating Community Mental Health Services in Scotland. Vol 2 (ed N. Drucker). Scottish Association for Mental Health.

KEHOE, R.F. \& MANDER, A.J. (1992) Lithium treatment: prescribing and monitoring habits in hospital and general practice. British Medical Journal, 304, 552-554.

*John R, Taylor, Registrar, Dingleton Hospital, Melrose, Borders Region TD6 9HN; and Ian G. Dewar, Registrar, Dingleton Hospital

*Correspondence 